Document downloaded from:

http://hdl.handle.net/10251/62418

This paper must be cited as:

García Breijo, E.; Prats Boluda, G.; Lidon-Roger, JV.; Ye Lin, Y.; Garcia Casado, FJ. (2015). A comparative analysis of printing techniques by using an active concentric ring electrode for bioelectrical

recording. Microelectronics International. 32(2):103-107. doi:10.1108/MI-03-2015-0021.

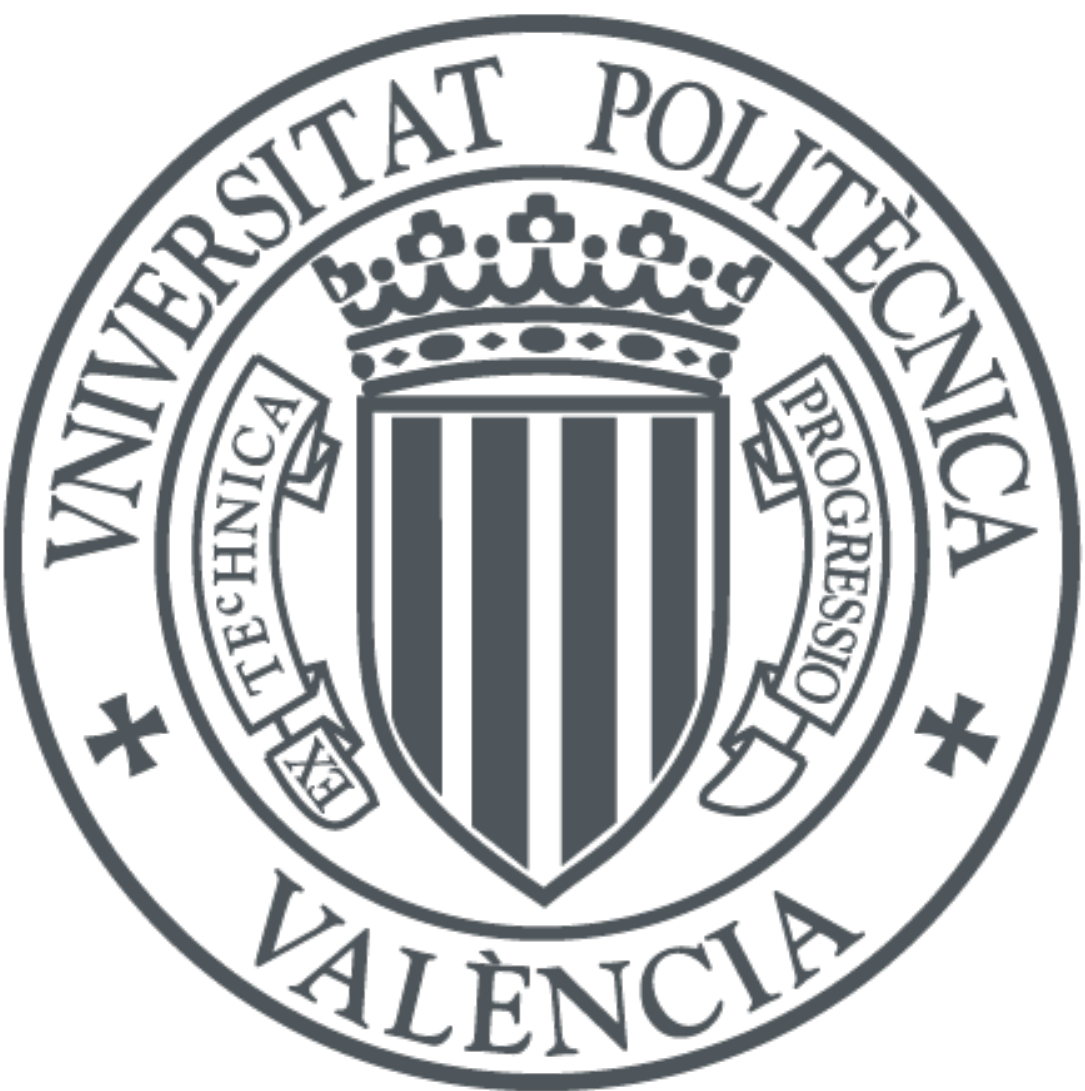

The final publication is available at

http://dx.doi.org/10.1108/MI-03-2015-0021

Copyright Emerald

Additional Information 


\title{
A comparative analysis of printing techniques by using an active concentric ring electrode for bioelectrical recording. ${ }^{1}$
}

\begin{abstract}
Purpose - This paper presents a comparison between three types of manufacturing techniques: screen-printed, inkjet and gravure, using different types of inks, for the implementation of concentric ring electrodes which permit estimation of laplacian potential on the body surface.

Design/methodology/approach - Flexible concentric ring electrodes not only present lower skin-electrode contact impedance and lower baseline wander than rigid electrodes but are also less sensitive to interference and motion artefacts. The above three techniques allow printing of conductive inks on flexible substrates and with this work we aim to study which is the best technique and ink to obtain the best electrode response.

Findings - From the results obtained regarding ink thickness, resistivity, electrode resistance, and other performance parameters derived from electrocardiographic signal recording tests: it can be said that concentric electrodes using the screen-printing and inkjet are suitable for noninvasive bioelectric signal acquisition.

Originality/value - The development of new types of inks and substrates for the electronics industry and the adaptation of new manufacturing techniques for allows an improvement in the development of electrodes and sensors.
\end{abstract}

Keywords Printed Electronics, Flexible Electrodes, Concentric ring electrodes, Bioelectrical recordings.

Paper type Research paper

\section{Introduction}

In the last decades, there has been a significant increase in the interest in using printing technologies as techniques for the development of low cost, large area electronic systems. These provide a wide range of advantages compared to traditional techniques of fabrication of silicon based electronics. One of the most important advantages is the possibility of working with flexible substrates such as plastic that has allowed the manufacture of sensors at low cost. The combination of additive fabrication techniques (inkjet, gravure, serigraphy, etc.) and roll-to-roll fabrication can lead to the production of ultra-low cost devices (FP7 Observatory, 2010) and what is more, it has allowed the development of new types of sensors with particularly relevant features for being used in bioengineering applications.

Regarding bioelectrical surface recordings, they are usually performed by unipolar or bipolar disc electrodes even though they entail the serious disadvantage of having poor spatial resolution. Laplacian potential on the body surface can be estimated by means of concentric ring electrodes (He and Cohen, 1992, Lu and Tarjan, 2002 and Besio et al, 2006). It has been proved that bioelectrical surface recordings performed with such concentric ring electrodes improve spatial resolution compared to unipolar or bipolar disc electrodes. However, concentric ring electrodes have commonly been implemented on rigid substrates. Therefore

\footnotetext{
1 This work was financially supported by the the Spanish Government and European FEDER funds (MAT2012-38429-C04-04)
} 
they do not adapt to the body surface curvature which provokes discomfort to the patient and a poor contact that affects the signal quality. An active sensor based on a tripolar concentric ring electrode in bipolar configuration (TCB) implemented using screen-printing technology on a flexible substrate for obtaining high spatial resolution ECG recording has already been developed. However in order to further improve the spatial resolution achieved with the TCB electrode, a tripolar concentric ring electrode (TCE) design is needed (Prats-Boluda et al, 2012).

At present, there are a several different printing techniques used in the fabrication of electronic devices, sensors or electrodes, which have their origin in graphic arts and can be used in the fabrication of these devices. The most widely used printing technologies in industry or research are screen printing, inkjet printing, stamping/nanoimprinting, and gravure printing. Obviously, there are many more techniques but they are not usually applied in the fabrication of electronic systems. It is worthwhile to highlight the advantages and disadvantages of each technique to determine which is the best technique in each application.

The screen-printing technology (Board, 2003) is the most used and mature printing technology. It has been used in the fabrication of electronic systems for decades. The revolution in the use of screen printing techniques on flexible substrates occurred with the development of polymer based inks which allow low curing temperatures compatible with plastic substrates.

Gravure printing (De la Fuente, 2009) is also a well-known technique that has its origin in the graphic arts. The ink is transferred from the gravure grooves to the substrate by means of a silicone rubber roller.

Inkjet printing (Magdassi, 2009) is an extensively used technique for printing electronic systems today. This technique allows the use of low viscosity inks; this is extremely important, since it allows for the formulation of polymer inks that only contain active material and solvents without the need for binders.

The aim of the present work, focused on the realization of a TCE, is to compare and find the best technology for manufacturing this kind of biomedical electrodes so as to obtain the best performance: exact geometry of the electrodes and capability for picking up noninvasively surface bioelectrical signals. Specifically the main printing technologies will be compared: screen, gravure and inkjet using different types of inks and substrates.

\section{Materials and methods}

The active sensor that will be used is made up of two parts: a disposable sensing TCE printed on a flexible substrate, and a reusable battery powered signal conditioning circuit that filters and preamplifies the biosignals before transmission. The sensing part consisted of two hook-shaped electrodes and an inner disc electrode (see Figure 1) so as to be implemented using a monolayer design (no bias are needed). The outer ring diameter was set to $36 \mathrm{~mm}$, which has been proven to successfully pick up tripolar Laplacian electrocardiogram (Besio, 2007). Other technical and physiological issues had been considered in the TCE design:

- $\quad$ The areas of the outer ring, the middle ring and the inner disc should be equal so as to provide similar electrode impedance.

- $\quad$ The inter electrode distance (D) between the inner disc and middle ring should be the same as the distance between the middle ring and the outer ring to obtain a tripolar Laplacian estimation, that is $D=r_{2 a}-r_{1}=r_{3 a}-r_{2 b}$; see Figure $1(a)$. 
Furthermore, issues such as a minimum recording area of $50 \mathrm{~mm}^{2}$ and a minimum ring width of $0.6 \mathrm{~mm}$ were also considered to ensure signal acquisition

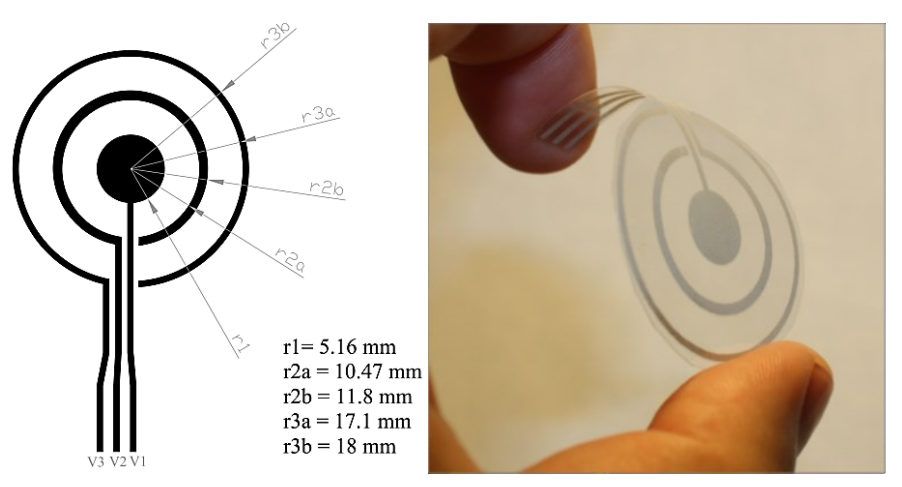

Figure 1 (a) Monolayer design of the TCE: V1, V2 and V3 are the biopotentials picked up by the inner disc, middle ring and outer ring respectively. (b) Photograph of an implemented flexible electrode

Tests were carried out in a previous work (Prats-Boluda et al, 2012) in both rigid PCB and flexible (Valox FR-1, Polyester Melinex ST506 and Ultem R16SG00) substrates to select the one that offered the best results concerning electrode flexibility, adhesion of the conductive and insulating pastes, and low skin-electrode impedance. Based on these tests, a MELINEX ST506 (175 $\mu \mathrm{m}$ of thickness, DuPont Teijin Films) substrate was chosen. MELINEX ST506 is a crystal clear, high gloss, heat stabilized Polyethylene terephthalate (PET) film.

Below there is a summary of three methods used in the present work for manufacturing the electrode. In each method, materials and equipment used are detailed. Finally, a comparison is shown in which the layer thickness obtained, the resistivity and resistance of one ring electrode is detailed. The layer thickness was measured with 190-00 HITEC Micrometer Digital, the resistivity was calculated by using ASTM F1896-10 conductivity test and measured Hewlett Packard HP34401 and normalized to a thickness of $25 \mu \mathrm{m}$, and the resistance of the ring was measured with a Keithley 2000 multimeter.

\subsection{Results and discussion}

\subsubsection{Screen printing Technology}

In order to develop the indicated electrode a screen was made with polyester material (textile) of 230 mesh, PET 1500 90/230-48W (Sefar AG, Switzerland). The main parameters of this material are a mesh-opening of $55 \mu \mathrm{m}$, open area of $24.6 \%$ and a mesh thickness of 78 $\mu \mathrm{m}$. A photo sensible film POLYCOL UNO (Kiwo GmbH, Deutschland), with a final layer thickness of $6 \mu \mathrm{m}$ after drying, was used with the mesh in order to obtain the electrode motif. The mask was exposure to 5000 W UV lamp for $2 \mathrm{~s}$.

The conductive inks used were three: Silver ink C2020522D1 (Gwent, UK), Nanoparticles Silver ink DGP-OS (Advanced Nano Product, Korea) and Carbon/Graphite C2030519P4 (Gwent, UK). The main characteristics of these inks are shown in Table I. 
The inks were serigraphed using a semiautomatic serigraphic machine AUREL 900 (Aurel, Italy). A $60^{\circ}$ Shore squeegee was selected. Printing speed was kept constant during the printing trials at around $10 \mathrm{~mm} / \mathrm{s}$ and a pressure of $2 \mathrm{~kg} \cdot \mathrm{cm}^{-2}$.

To check the accuracy obtained after printing the size difference between a line and the printed artwork was determined. A line in the photo mask was designed with $0.88 \mathrm{~mm}$ of width and the width of the line obtained in the screen was $0.88 \mathrm{~mm}$. The width of the conductor track obtained after making the serigraphy was $0.9 \mathrm{~mm}$, therefore a $2.2 \%$ error due to the impression was obtained.

The thickness of the conductor before drying was calculated using the equation (1).

$$
\mathrm{T}_{\mathrm{bd}}=\left(\mathrm{T}_{\mathrm{s}} \cdot \mathrm{A}_{\mathrm{s}}\right)+\mathrm{T}_{\mathrm{f}}=(78 \mu \cdot 0.24)+6 \mu=24.72 \mu \mathrm{m}
$$

Where $T_{b d}$ is the conductor thickness before drying, $T_{S}$ is the screen thickness, $A_{S}$ is the open area of screen and $T_{f}$ is the photo sensible film thickness. $T_{b d}$ will be reduced up to $60 \%$ (it is depends on the solids contents) after drying so the final thickness should be around $15 \mu \mathrm{m}$.

A study of the final thickness of the inks according to the curing temperature recommended by the manufacturer $\left(60\right.$ to $\left.150^{\circ} \mathrm{C}\right)$ was performed. The track thickness with C2020522D1 and C2030519P4 inks was around $14 \mu \mathrm{m}$, being nearly the calculated thickness in Equation1, but the thickness for the DGP-OS is around $8 \mu \mathrm{m}$, clearly under $15 \mu \mathrm{m}$. It could be due to the use of nanoparticles in its composition and, therefore resulting in different rheological and thixotropical characteristics.

\subsubsection{Gravure printing Technology}

In order to build the indicated electrode plate, with a line density of $120 \mathrm{I} \cdot \mathrm{cm}-1$ laser engraving and a cell depth of $25 \mu \mathrm{m}$, was used.

The two conductive inks utilized were: Silver flexographic ink C2080815P1 (Gwent, UK) and Carbon flexographic C2080529D7 (Gwent, UK). The main characteristics of these inks are shown in Table I.

The inks were deposited on the top of the substrate using a laboratory scale gravure press K-Printing Proofer (Testing Machines Inc., USA). A Doctor Blade with an angle of $45^{\circ}$ was used. The speed of the ink doctoring on the gravure was about $8 \mathrm{~m} / \mathrm{min}$ to allow the ink to be doctored with sufficient doctoring pressure. A $65^{\circ}$ Shore cylinder was selected.

To check the accuracy obtained after printing the size difference between a line and the printed artwork was measured. A line in the pattern was designed with $0.88 \mathrm{~mm}$ of width and the width of the line obtained in the plate after fabrication was $1.1 \mathrm{~mm}$. The width of the conductor track obtained after making the gravure was $1.1 \mathrm{~mm}$; therefore a $20 \%$ error due to the plate making was obtained.

In gravure printing, it is difficult to calculate the final thickness of the conductor since it depends on many factors such as cell depth, ink viscosity, speed of engraving, type of substrate, etc. A study of the final thickness of the inks according to the curing temperature recommended by the manufacturer (60 to 150 ㅇ C) was performed. The $\mathrm{Ag} / \mathrm{C} 2080815 \mathrm{P} 1$ average thickness is $4 \mu \mathrm{m}$ while the $\mathrm{C} / \mathrm{C} 2080529 \mathrm{D} 7$ is $3.8 \mu \mathrm{m}$.

\subsubsection{Inkjet printing Technology}


A Dimatix Materials DMP-2800 (FujiFilm, USA), based on piezo jetting technology was used as the patterning instrument [4]. This printer is based on a cartridge print-head system. Fluid was injected into the fluid module. The fluid module was then attached to the jetting module to form a sealed cartridge. The inkjet print head itself consists of a silicon die with sixteen individually addressable jets which are spaced $254 \mu \mathrm{m}$ apart. The effective nozzle diameter is $21.5 \mu \mathrm{m}$, which provide droplets that are $10 \mathrm{pL}$ in volume. The waveform pulse shape, amplitude, slew rate and duration, frequency and voltage were optimized for each ink independently. The differences between both were: Jetting frequency of $8 \mathrm{kHz}$ for EMD5603 and $5 \mathrm{kHz}$ for DGP40LT-15C and 4 jets for EMD5603 and 16 jest for DGP4OLT-15C.

Two conductive inks were used: Nanoparticles Silver ink EMD5603 (SunChemical, UK), Nanoparticles Silver ink DGP40LT-15C (Advanced Nano Product, Korea). The main characteristics of these inks are shown in Table I.

EMD5603 is a solvent based ink so a low cartridge temperature was used but DGP40LT-15 is an aqueous based ink so a high cartridge temperature was used; $40^{\circ} \mathrm{C}$ forDGP40LT-15C and 28ㅇ C for EMD5603.

The pattern was drawn with the computer programme adjoined to the printer with 600 dpi of resolution. The inkjet printing was done as horizontal, bar-by-bar printing, using a DMC11610.

The EMD5603 ink showed good adherence over the substrate but there was an adherence trouble with the DGP4OLT-15 ink. In this case, a pre-treatment of the substrate surface was done; an $80^{\circ} \mathrm{C} / 10$ min heating was applied.

\begin{tabular}{|c|c|c|c|c|c|c|c|}
\hline Property & $\begin{array}{c}\mathrm{Ag} \\
\mathrm{C2020522D1}\end{array}$ & $\begin{array}{c}\mathrm{Ag} \\
\text { DGP-OS }\end{array}$ & $\begin{array}{c}C \\
\text { C2030519P4 }\end{array}$ & $\begin{array}{c}A g \\
C 2080815 P 1\end{array}$ & $\begin{array}{c}C \\
\text { C2080529D7 }\end{array}$ & $\begin{array}{c}\mathrm{Ag} \\
\text { EMD5603 }\end{array}$ & $\begin{array}{c}\text { Ag DGP40LT- } \\
15 \mathrm{C}\end{array}$ \\
\hline $\begin{array}{l}\text { Solids Contents } \\
\text { [\%] }\end{array}$ & $77 \sim 79$ & $70 \sim 80$ & $39 \sim 43$ & 67 & 32 & 20 & $30 \sim 35$ \\
\hline Viscosity [Pa.s] & $18 \sim 20$ & $100 \sim 300$ & $3.1 \sim 4.8$ & $0.8 \sim 1.0$ & $0.2 \sim 0.5$ & $10 \sim 13$ & $10 \sim 17$ \\
\hline $\begin{array}{l}\text { Specific } \\
\text { Resistivity } \\
{[\Omega \cdot \mathrm{cm}]}\end{array}$ & $\cdot$ & $10 \times 10^{-6}$ & - & $6.5 \times 10^{-13}$ & $1.09 \times 10^{-2}$ & $27 \sim 31$ & $35 \sim 38$ \\
\hline
\end{tabular}

To check the printing accuracy obtained after printing the size difference between a line and the printed artwork was determined. A line in the pattern was designed with $0.88 \mathrm{~mm}$ of width and the width of the conductor track obtained after making the printing was $1.2 \mathrm{~mm}$, which corresponds to a $26.6 \%$ error due to printing.

In inkjet printing, it is also difficult to calculate the final thickness of the conductor since it depends on many factors such as ink viscosity, ink drying, etc. A study of the final thickness of the inks according to the curing temperature recommended by the manufacturer (120 to 200 C) was performed. The final thickness was around $3 \mu \mathrm{m}$ in both inks.

\section{Results}


The resistivity was, in the case of screen-printed inks: $57.2 \Omega / \mathrm{sq}$ for C2030519P4, 0.058 $\Omega / \mathrm{sq}$ for DGP-OS and $0.024 \Omega / \mathrm{sq}$ for C2020522D1; and the resistance of the outer ring is $13,400 \Omega$ with C2030519P4, $24.44 \Omega$ with DGP-OS and $6.76 \Omega$ with C2020522D1.

In the case of gravure inks the resistivity was: $1.42 \Omega /$ sq for C2080815P1 and $2.94 \Omega /$ sq for C2080529D7; and the resistance of the outer ring is 1,380 $\Omega$ with C2080815P1 and 283,000 $\Omega$ with C2080529D7. A difference between the expected and the obtained resistivity, of more than 8 times is observed in the case of C2080529D7. This may be due to a spread in the ink pattern which makes the resistivity higher than what can be expected according to the data sheet of manufacturer. This problem is not attributable to the limits of the gravure technology since better results would be obtained by changing the line density and the cell depth; but the inconvenient in improving those results is the high cost of the gravure plates. Moreover, an investment in better gravure plates does not mean better results compared with the other techniques.

Finally, in the case of inkjet inks the resistivity was: $0.03 \Omega / \mathrm{sq}$ for EMD5603 and $0.018 \Omega / \mathrm{sq}$ for DGP40LT-15C; and the resistance of the outer ring is $30 \Omega$ with EMD5603 and $46 \Omega$ with DGP40LT-15C.

Table II shows a summary of the average and standard deviation for six samples of the thickness, resistivity and resistance of the outer ring for the various inks used. In non-invasive biomedical signal recordings, it is essential to ensure skin-electrode contact impedance is as low as possible, and also therefore the resistance of the electrode rings. In this sense the electrodes obtained with screen printing and inkjet have similar resistance and less than $50 \Omega$, while gravure technique yielded resistances much higher and is out of the range suitable to pick up non-invasively bioelectric signals of small amplitude (hundred microvolts to millivolts) so its use was discarded in the next phases of this work. As stated before this does not mean that gravure cannot get a track resistance similar to other printing techniques but, in this case, this would require making a new plate with engraving cost increasing disproportionately. Electrodes made with the inkjet technique presented smaller ink thickness than those obtained with screen printing, which slightly increases the resistance of the electrodes.

TABLE II . Summary of the average and standard deviation of the thickness, resistivity and resistance of the outer ring for the various inks used

\begin{tabular}{|c|c|c|c|c|c|c|c|}
\hline & \multicolumn{3}{|c|}{ Screen-printing } & \multicolumn{2}{|c|}{ Gravure } & \multicolumn{2}{|c|}{ Inkjet } \\
\hline & DGP-OS & C2030519P4 & C2020522D1 & C2080815P1 & C2080529D7 & EMD5603 & $\begin{array}{l}\text { DGP40LT- } \\
15 \mathrm{C}\end{array}$ \\
\hline $\begin{array}{l}\text { Thickness } \\
{[\mu \mathrm{m}]}\end{array}$ & $8.63 \pm 3.91$ & $15.5 \pm 1$ & $12.8 \pm 1.5$ & $4.9 \pm 0.8$ & $3.8 \pm 1.4$ & $3.83 \pm 2.78$ & $1.58 \pm 0.45$ \\
\hline $\begin{array}{l}\text { Resistivity } \\
{[\Omega / s q]}\end{array}$ & $0.048 \pm 0.013$ & $57.2 \pm 13.2$ & $0.024 \pm 0.003$ & $1.42 \pm 0.55$ & $2,940 \pm 1,113$ & $0.03 \pm 0.02$ & $0.018 \pm 0.002$ \\
\hline $\begin{array}{l}\text { Resistance } \\
\text { External } \\
\text { ring }[\Omega]\end{array}$ & $24.44 \pm 0.01$ & $13,400 \pm 2,240$ & $6.76 \pm 0.08$ & $1,380 \pm 793$ & $283,000 \pm 3,920$ & $30 \pm 2$ & $46 \pm 13$ \\
\hline
\end{tabular}

\section{Discussion}

So as to assess the detectability and signal quality of bioelectrical signals picked up by screen printing vs inkjet TCR electrodes, ECG recordings were carried out and various quantitative parameters relating to the detectability and signal quality were obtained: skin contact electrode impedance, amplitude of the ECG signal (Vsp-p) and signal-to-noise ratio (SNR); see Tables III and IV. Specifically C2020522D1 paste and EMD5603 ink electrodes were 
used in this comparison due to the fact that they provided the lowest resistance of the external ring for each technology.

Twenty six recording sessions were carried out on healthy volunteers ( 8 women and 18 men); mean age was $32.6 \pm 16.2$ years (range 16-74) and mean body mass index was $24.6 \pm 4.9$ $\mathrm{kg} / \mathrm{m} 2$. The study adhered to the standards of the Declaration of Helsinki. The volunteers were informed of the nature of the study, briefed on the recording protocol and signed a consent form. The subjects were sitting during the recordings and were asked to relax and remain so throughout the session to avoid the influence of fluctuation of heart position on the body surface ECG, but they were free to talk. The skin area on which the electrodes were placed was previously exfoliated and, in the case of male volunteers, was also shaved. A TCE (inkjet or serigraphy) was placed $6 \mathrm{~cm}$ above and $2 \mathrm{~cm}$ to the right of the left nipple (upper position), the analog ground being connected to a monopolar disposable external reference electrode located on the right hip. After 5 minutes, skin-electrode contact impedance of each electrode pole was measured using the General Devices EIM 105 impedance meter, which is based on three electrode impedance measurement techniques. For this, two additional disposable electrodes were placed on both shoulders of the subjects. Subsequently 5 minutes of ECG signals picked up with the TCE were recorded. Then the TCE electrode was removed, skin cleaned, the other type of TCE (inkjet or serigraphy) was placed in the same position and the previous protocol was repeated. In order to compare the results, 13 of the 26 sessions started using screen printing electrodes while the remaining 13 used inkjet electrodes first.

The potential difference sensed by the TCE's outer ring electrode and the inner disc (BCb) and that of the middle ring and the inner disc ( $\mathrm{BCa}$ ) was worked out by an analogue conditioning circuit directly connected to the TCE electrode. The conditioned signals were simultaneously acquired using a 16 bit data acquisition card with a sampling rate of $1 \mathrm{kHz}$.

\begin{tabular}{lccc}
\multicolumn{2}{l}{ Table III.- Skin-electrode impedance $(\mathrm{k} \Omega)$} & & \\
\cline { 2 - 2 } & Inner disc & Middle ring & Outer ring \\
\hline Inkjet printing & $2.40 \pm 1.24$ & $2.18 \pm 0.74$ & $2.46 \pm 1.01$ \\
Screen-printing & $2.43 \pm 0.807$ & $2.54 \pm 0.86$ & $3.43 \pm 1.22$ \\
\hline \hline
\end{tabular}

The results in Table IV show no statistically significant differences between the electrodes implemented with inkjet or screen printed electrodes, in terms of the ECG signal amplitude and signal to noise ratio.

Table IV.- Average and standard deviation of the quantitative parameters of the ECG signals acquired with the TCE located in the chest. $\mathrm{BCa}$ is the differential signal between the intermediate ring and central disk; $\mathrm{BCb}$ is the differential signal between the outer ring and the central disk.

\begin{tabular}{|c|c|c|c|c|}
\hline & \multicolumn{2}{|c|}{$\mathrm{BCa}$} & \multicolumn{2}{|c|}{ BCb } \\
\hline & Vsp-p $(\mu \mathrm{V})$ & SNR (dB) & Vsp-p $(\mu \mathrm{V})$ & SNR (dB) \\
\hline Inkjet printing & $83.7 \pm 26.1$ & $15.8 \pm 3.3$ & $138.6 \pm 40.9$ & $18.4 \pm 2.9$ \\
\hline
\end{tabular}

\section{Conclusions}

Although considered a mature technology, screen-printing still provides good results in the generation of printed electrodes. In this paper we have obtained the characteristics of a TCE manufactured used inkjet, gravure and screen-printing technologies. ECG signals have been obtained using inkjet and screen printed TCE without significant differences in terms of ECG signal amplitude and signal to noise ratio. Gravure TCE was discarded due to the high values of 
resistance obtained. From these results, it can be seen that TCE fabrication using the screenprinting and inkjet printing are both suitable for non-invasive bioelectric signal acquisition. However, screen-printing is preferred due to its lower cost and production time.

\section{References}

Besio, W. and Chen, T. "Tripolar Laplacian electrocardiogram and moment of activation isochronal mapping," Physiol Meas., vol. 28, no. 5, pp. 515-529, May2007

Besio, W., Aakula, R., Koka, K. and Dai, W. "Development of a tri-polar concentric ring electrode for acquiring accurate Laplacian body surface potentials," Ann. Biomed. Eng, vol. 34, no. 3, pp. 426-435, Mar.2006.

Board, N. Screen Printing Technology Hand Book. National Institute Of Industria. 2003.

De la Fuente Vornbrock, A. Roll Printed Electronics: Development and Scaling of Gravure Printing Techniques. Electrical Engineering and Computer Sciences, University of California at Berkeley, Technical Report No. UCB/EECS-2009-191.

He, B. and Cohen, R. J. "Body surface Laplacian mapping of cardiac electrical activity," Am. J. Cardiol., vol. 70, no. 20, pp. 1617-1620, Dec.1992

ICT Sector Focus Report. Printed Electronics (FP7 Observatory NANO European Nanotechnology , 2010).

Lu, C.C. and Tarjan, P.P. "An ultra-high common-mode rejection ratio (CMRR) AC instrumentation amplifier for laplacian electrocardiographic measurement," Biomed. Instrum. Technol., vol. 33, no. 1, pp. 76-83, 2002.

Magdassi, S. The Chemistry of Inkjet Inks. The Hebrew University of Jerusalem, Israel. 2009.

Prats-Boluda, G., Ye-Lin, Y., Garcia-Breijo, E., Ibanez, J. and Garcia-Casado, J. "Active flexible concentric ring electrode for non-invasive surface bioelectrical recordings," Measurement Science \& Technology, vol. 23, no. 122012. 